\title{
Anxiety and Depression in Patients with Inflammatory Bowel Diseases: The First Step toward Proper Management
}

\author{
Jung Won Lee \\ Department of Internal Medicine, Samsung Changwon Hospital, Sungkyunkwan University School of Medicine, Changwon, Korea
}

\begin{abstract}
See "Unmet Psychosocial Needs of Patients with Newly Diagnosed Ulcerative Colitis: Results from the Nationwide Prospective Cohort Study in Korea" by Jung Rock Moon, et al. on page 459, Vol. 14, No. 4, 2020
\end{abstract}

Inflammatory bowel diseases (IBDs) are known to cause chronic intestinal inflammation and deteriorate patient quality of life in various ways. ${ }^{1,2}$ IBDs have unique features that cannot be easily managed, and most patients have exacerbated disease courses and complications over time. ${ }^{2}$ The long-lasting, fluctuating, and often severe disease courses of IBDs can cause various psychiatric problems. Ulcerative colitis (UC) is often accompanied by unpredictably frequent bowel movements and bloody stools, which can cause fear of not having immediate access to a toilet. ${ }^{1}$ This is regarded to be the underlying mechanism of anxiety and depression in patients, especially for those with uncontrolled UC. Therefore, patients with significant IBDs have been confirmed to develop anxiety and depression in various cohort studies. Moreover, many studies have also shown that when poorly controlled, patient anxiety and depression can lead to frequent recurrence of IBDs, ${ }^{3}$ increased hospitalization rates, ${ }^{4}$ and reduced treatment compliance. ${ }^{5}$

Despite the high incidence of anxiety and depression along with poor quality of life in patients with IBDs, screening and treatment for psychiatric complications have been often underestimated. In addition to improving the disease activity in patients with IBDs, the demand for observation and improvement of the overall patient quality of life has been a long-standing problem. As patients reported outcomes have become important indexes of IBDs, ${ }^{6}$ several published cohort studies have focused on psychiatric problems and assessed patients using the Hospital Anxiety and Depression Scale (HADS), Short-Form 12 (SF-12), and Inflammatory Bowel Disease Quality-of-Life Questionnaire (IBDQ). However, the study by Moon et al. ${ }^{7}$ appears to be the first to comprehensively quantify psychiatric problems using the HADS, Work Productivity and Activity Impairment (WPAI), SF-12, and IBDQ, simultaneously. In this study, severe loss of work productivity and social activity was reported at $46.2 \%$ and 53.5\%. The prevalence of comorbid psychiatric illnesses, defined as a HADS score $\geq 8$, among the patients was 33.7\% for anxiety and $41.8 \%$ for depression respectively. In addition, significant mood disorders requiring psychological interventions, defined by a HADS score $\geq 11$, were identified in 16.7\% (anxiety) and 20.6\% (depression) of patients. Furthermore, work activity impairment was significantly higher in patients with severe disease activity than in those with moderate disease activity (all $\mathrm{p}<0.05$ ). These data clearly identified recent unmet psychosocial needs among newly diagnosed patients with UC in Korea.

South Korea is currently experiencing a steep increase in the incidence of IBDs compared with Western countries. The first systematic nationwide cohort study of UC was launched in 2014 in South Korea. This ongoing prospective cohort study only includes patients with newly diagnosed moderate-to-severe UC and is called the moderate-to-severe ulcerative colitis in Korea (MOSAIK) cohort study (Clinicaltrials.gov, NCT02229344). ${ }^{8}$ The ongoing study aims to capture all eligible patients with newly diagnosed UC in referred centers. The first patient consented to participate in the study in August 2014, and as of March 2017, the study has enrolled 355 patients. The study will release a report on major findings in February 2023. The MOSAIK cohort study has unique strengths in terms of the comprehensive outcome of IBD care and intervention to improve quality of life because no studies have collected the HADS, WPAI, SF-12, and IBDQ simultaneously.

The present study strongly proposes a need for point-of-care

Correspondence to: Jung Won Lee

Division of Gastroenterology, Samsung Changwon Hospital, Sungkyunkwan University School of Medicine, 158 Paryong-ro, Masanhoewon-gu, Changwon 51353, Korea

Tel: +82-55-233-5000, Fax: +82-55-233-5028, E-mail: saludos@naver.com

pISSN 1976-2283 eISSN 2005-1212 https://doi.org/10.5009/gnl20187

(c) This is an Open Access article distributed under the terms of the Creative Commons Attribution Non-Commercial License (http://creativecommons.org/licenses/by-nc/4.0) which permits unrestricted non-commercial use, distribution, and reproduction in any medium, provided the original work is properly cited. 
screening and timely interventions for psychosocial distress among patients with newly diagnosed UC, particularly those with moderate-to-severe UC. Unfortunately, no specific study plan has been proposed for providing timely intervention and care. Prospective studies are rarely conducted to investigate whether adequate psychiatric interventions may be helpful in patients experiencing anxiety or depression. Moreover, it is important to recognize that gastroenterologists are not always experts in all manifestations of IBDs. Therefore, it is important to conduct a prospective study that includes experts who can effectively manage patients' psychiatric problems. However, the disease flare and exacerbation of psychiatric symptoms has been proved to be proportional in similar studies. Therefore, proper control over disease activity could also improve the psychiatric symptoms in patients with IBD. Unfortunately, no study has shown how effective control of psychiatric symptoms can independently alleviate IBD outcomes. In particular, with the recent introduction of new biologics, future challenges should be explored to determine whether adequate psychiatric intervention can independently improve patient outcomes, especially on the impact on hospitalization, recurrence rate, and treatment compliance.

\section{CONFLICTS OF INTEREST}

No potential conflict of interest relevant to this article was reported.

\section{ORCID}

Jung Won Lee

https://orcid.org/0000-0002-7945-1618

\section{REFERENCES}

1. Yarlas A, Rubin DT, Panés J, et al. Burden of ulcerative colitis on functioning and well-being: a systematic literature review of the SF-36 ${ }^{\circledR}$ health survey. J Crohns Colitis 2018;12:600-609.

2. Panara AJ, Yarur AJ, Rieders B, et al. The incidence and risk factors for developing depression after being diagnosed with inflammatory bowel disease: a cohort study. Aliment Pharmacol Ther 2014;39:802-810.

3. Mittermaier C, Dejaco C, Waldhoer T, et al. Impact of depressive mood on relapse in patients with inflammatory bowel disease: a prospective 18-month follow-up study. Psychosom Med 2004;66:79-84.

4. van Langenberg DR, Lange K, Hetzel DJ, Holtmann GJ, Andrews JM. Adverse clinical phenotype in inflammatory bowel disease: a cross sectional study identifying factors potentially amenable to change. J Gastroenterol Hepatol 2010;25:1250-1258.

5. Nigro G, Angelini G, Grosso SB, Caula G, Sategna-Guidetti C. Psychiatric predictors of noncompliance in inflammatory bowel disease: psychiatry and compliance. J Clin Gastroenterol 2001;32:6668.

6. IsHak WW, Pan D, Steiner AJ, et al. Patient-reported outcomes of quality of life, functioning, and GI/psychiatric symptom severity in patients with inflammatory bowel disease (IBD). Inflamm Bowel Dis 2017;23:798-803.

7. Moon JR, Lee CK, Hong SN, et al. Unmet psychosocial needs of patients with newly diagnosed ulcerative colitis: results from the nationwide prospective cohort study in Korea. Gut Liver 2020;14:459-467.

8. Lee CK, Lee KM, Park DI, et al. A new opportunity for innovative inflammatory bowel disease research: the moderate-to-severe ulcerative colitis in Korea (MOSAIK) cohort study. Intest Res 2019;17:1-5 